\title{
Editorial
}

\section{Introduction of the special issue on the 10th WESTPAC International Scientific Conference}

\author{
Fangli Qiao ${ }^{1,2,3 *}$, Si Tuan $\mathrm{Vo}^{4}$, Wenxi $\mathrm{Zhu}^{5}$ \\ ${ }^{1}$ First Institute of Oceanography, Ministry of Natural Resources, Qingdao 266061, China \\ ${ }^{2}$ Laboratory for Regional Oceanography and Numerical Modeling, Pilot National Laboratory for Marine Science and \\ Technology (Qingdao), Qingdao 266237, China \\ ${ }^{3}$ Key Laboratory of Marine Science and Numerical Modeling, Ministry of Natural Resources, Qingdao 266061, China \\ ${ }^{4}$ Institute of Oceanography, Vietnam Academy of Science and Technology, Nha Trang 57129, Vietnam \\ ${ }^{5}$ IOC Sub-Commission for the Western Pacific, Intergovernmental Oceanographic Commission of UNESCO, Bangkok \\ 11120, Thailand
}

Received 3 January 2019; accepted 6 January 2019

(c) Chinese Society for Oceanography and Springer-Verlag GmbH Germany, part of Springer Nature 2019

This special issue is the scientific legacy of the 10th WESTPAC International Scientific Conference, which was sponsored by State Oceanic Administration of China and the Intergovernmental Oceanographic Commission of UNESCO, and organized by the IOC SubCommission for the West Pacific (WESTPAC) and the First Institute of Oceanography of China, which was held in Qingdao, China during 17-20 April 2017 with more than 700 participants from 21 countries. During the conference, the international scientific steering committee announced the special issue of Acta Oceanologica Sinica for building a scientific legacy. Forty-five manuscripts were submitted with 20 papers accepted for this special issue.

In this special issue, Afifi and Fadzil explored the thermocline and water masses variability during 1951 and 2014 in the southern South China Sea; Daud et al. detected the influence of ENSO on the upwelling and oceanic temperature fronts off Malaysia which are important for fishery production in this area; Guo et al. analyzed the Kurushio intrusion into the South China Sea by using observed Argos drifters from 1988 to 2012, and suggested that the sea level pressure plays key role for this intrusion; Kashem et al. analyzed the response of 8 oceanic parameters during tropical cyclone Viyaru in 2013 over the bay of Bengal which may help the forecasting ability of Typhoon; Rosali and Fadzil reported the oceanic temperature variability induced by tidal mixing off east coast of Peninsular Malaysia based on three years observations; Xia et al. simulated the summer circulation in the Bohai Sea based on a high-resolution wave-tide-circulation coupled model; Yin et al. developed the first jellyfish model which can be used to guide the stock enhancement; The accurate seasonal prediction of climate system has been a long-standing problem; Zhao et al. showed that the prediction skill of FIO-ESM is dramatically improved by $23.6 \%$ (11.6\%) over the Climate Forecast System Version 2 for ENSO and NPV out of phase (in phase) at initial conditions; Afdal determined the origin of the suspended particulate matter in the seagrass meadow by using a stable isotope signature; Du et al. evaluated the performance of 4 conventional fusion methods for estimating inland water body from satellite images, and concluded that Gram-Schmidt should be the best solution; Li et al. examined the relationship among phytoplankton size structure and environmental variables and zooplankton community structure in Changjiang Estuary both in spring and autumn; Jiang et al. analyzed the interesting echolocation signals from fleeing Irrawaddy dolphins; Nguyen and Jutta used microsatellite markers to assess the genetic diversity and population structure of 6 distinct seagrass beds and reported that the genetic diversity in the open sea is higher than that in the lagoon; Pengsakun et al. analyzed two coral bleaching events due to ocean temperature and flooding respectively; while Samsuvan et al. assessed the coral health; By using quantitative data from 19 sites, Vo et al. studied the resilience of coral reefs against increased ocean temperature and anthropogenic impact which can benefit the oceanic ecological management; Wang et al. isolated 48 and 34 cultivable bacterial strains from laboratory samples and suggested that bacteria associated with C. marina may have ecological roles in its competition with diatoms; Gao et al. documented the assemblage of dinoflagellate cyst on island in the East China Sea; Tang et al. used data from 4 seamounts in the West Pacific to study geochemistry and ages. We believe all above interesting results will be attractive for oceanographers, oceanic governors, young scholars and PhD candidates.

The contents of all articles in this volume lie under the sole responsibility of their authors. The designations employed and the presentation of the material in this publication do not imply the expression of any opinion whatsoever of the conference organizers or authors of this note concerning the legal status of any country, territory, city or area or of its authorities, or concerning the delimitation, and the naming of its frontiers.

Foundation item: The NSFC-Shandong Joint Fund for Marine Science Research Centers under contract No. U1606405; the International Ccooperation Project of Indo-Pacific Ocean Environment Variation and Air-Sea Interaction under contract No. GASIIPOVAI-05; the IOC/WESTPAC OFS Project.

*Corresponding author, E-mail: qiaofl@fio.org.cn 